\title{
Superficial Bound of the Depth Limit of Two- Photon Imaging in Mouse Brain
}

\author{
Kevin Takasaki, Reza Abbasi-Asl, and $\mathbb{D}$ Jack Waters
}

https://doi.org/10.1523/ENEURO.0255-19.2019

Allen Institute for Brain Science, Seattle, WA 98109

\begin{abstract}
Two-photon fluorescence microscopy has been used extensively to probe the structure and functions of cells in living biological tissue. Two-photon excitation generates fluorescence from the focal plane, but also from outside the focal plane, with out-of-focus fluorescence increasing as the focus is pushed deeper into tissue. It has been postulated that the two-photon depth limit, beyond which results become inaccurate, is where in-focus and out-of-focus fluorescence are equal, which we term the balance depth. Calculations suggest that the balance depth should be at $\sim 600 \mu \mathrm{m}$ in mouse cortex. Neither the two-photon depth limit nor the balance depth have been measured in brain tissue. We found the depth limit and balance depth of two-photon excitation in mice with GCaMP6 indicator expression in all layers of visual cortex, by comparing near-simultaneous two-photon and three-photon excitation. Two-photon and three-photon results from superficial locations were almost identical. two-photon results were inaccurate beyond the balance depth, consistent with the depth limit matching the balance depth for two-photon excitation. However, the two-photon depth limit and balance depth were at 450 $\mu \mathrm{m}$, shallower than predicted by calculations. Our results were from tissue with a largely homogenous distribution of fluorophores. The expected balance depth is deeper in tissue with fewer fluorophores outside the focal plane and our results therefore establish a superficial bound on the two-photon depth limit in mouse visual cortex.
\end{abstract}

Key words: cortex; 2-photon; 3-photon; multiphoton; fluorescence

\section{Significance Statement}

This study measures the maximum depth in the mouse brain to which it is possible to obtain quantitatively accurate results with two-photon microscopy, a form of non-linear fluorescence microscopy.

\section{Introduction}

Two-photon excitation permits fluorescence imaging with cellular and subcellular resolution hundreds of mi-

Received July 2, 2019; accepted December 2, 2019; First published January 6, 2020.

The authors declare no competing financial interests.

Author contributions: K.T., R.A.-A., and J.W. designed research; K.T. performed research; K.T., R.A.-A., and J.W. analyzed data; K.T., R.A.-A., and J.W. wrote the paper.

This work was supported by the Allen Institute for Brain Science.

Acknowledgements: We thank the Allen Institute founder, Paul G. Allen, for his vision, encouragement, and support. We also thank members of the Research Engineering and Neural Coding teams for helpful discussions and Ariel Leon for hardware support

Correspondence should be addressed to Kevin Takasaki at kevint@alleninstitute.org or Jack Waters at jackw@alleninstitute.org.

https://doi.org/10.1523/ENEURO.0255-19.2019

Copyright (C) 2020 Takasaki et al. crometers into biological tissue. Generally, the maximal imaging depth (depth limit) of two-photon excitation is determined by fluorescence from outside the focal plane. As the focal plane is pushed deeper into scattering tissue, illumination intensity at the tissue surface must be increased to maintain intensity in the focal plane, resulting in an increase in out-of-focus fluorescence with increasing depth (Ying et al., 1999; Theer et al., 2003). In a seminal study, Theer and Denk (2006) explored twophoton excitation analytically, calculating the expected in-focus and out-of-focus fluorescence under different conditions, including at different depths. Theer and Denk

This is an open-access article distributed under the terms of the Creative Commons Attribution 4.0 International license, which permits unrestricted use, distribution and reproduction in any medium provided that the original work is properly attributed. 
(2006) arbitrarily identified the two-photon depth limit as the depth at which the detected fluorescence generated by ballistic and scattered excitation light outside the focal plane equals that from fluorophores excited in the ballistic focus. The ratio of in-focus and out-of-focus fluorescence is a complex function of numerous factors, including numerical aperture, laser pulse duration, scattering anisotropy, and fluorophore distribution, but the calculations of Theer and Denk (2006) suggest that in-focus and out-offocus fluorescence are equal at approximately three scattering length constants under typical imaging conditions. In rodent cortical gray matter, three scattering length constants corresponds to $\sim 600 \mu \mathrm{m}$ below the tissue surface.

Three-photon excitation permits deeper imaging than two-photon excitation, in part because three-photon excitation generates fluorescence almost exclusively from the focal plane (Kobat et al., 2009, 2011; Horton et al., 2013; Ouzounov et al., 2017; Yildirim et al., 2019). In the absence of out-of-focus fluorescence, one expects the functional properties of neurons measured with twophoton and three-photon excitation to be identical, but the impact of out-of-focus fluorescence has not been measured. three-photon excitation offers the opportunity to estimate in-focus and out-of-focus fluorescence and thereby test the predictions of earlier analyses. We implemented near-simultaneous two-photon and three-photon excitation to compare results 200-650 $\mu \mathrm{m}$ below the surface of the brain in transgenic mice with dense GCaMP6 expression throughout neocortex. Our results indicate that two-photon and three-photon excitation produce equivalent results in superficial layers but not in deep in cortex, that the depth limit of two-photon excitation is where in-focus and out-of-focus fluorescence are equal, and that this depth is $450 \mu \mathrm{m}$.

\section{Materials and Methods}

\section{Basic three-photon microscope}

Our three-photon microscope was built around a Coherent Monaco/Opera-F laser source ( $\leq 2 \mathrm{~nJ}, 50$ fs pulses at $1 \mathrm{MHz}$; Coherent Inc.) and a modified MIMMS microscope manufactured by Sutter Instrument. We replaced the scan and tube lenses (respectively, Thorlabs SL50-3P and a Plössl pair of achromatic doublets, Thorlabs AC254-400-C) to improve transmission at $1300 \mathrm{~nm}$. The primary dichroic mirror was FF735-DI02 (Semrock). We used an Olympus $25 \times / 1.05$ objective $(75 \%$ transmission at $1300 \mathrm{~nm}$ ) or Nikon $16 \times / 0.8$ objective $(50 \%$ transmission at $1300 \mathrm{~nm}$ ) and image acquisition was controlled by Scanlmage (Vidrio Technologies LLC) with acquisition gating for low rep rate lasers.

We estimated group delay dispersion (GDD) through the microscope at $\sim 15,000 \mathrm{fs}^{2}$, approximately half of which was attributable to the Pockels cell (360-40-03LTA, Conoptics Inc). To compensate, we built a four-pass pulse compressor using a single SF-11 glass prism (Thorlabs PS-853) and a two hollow roof prism mirrors (Thorlabs HRS1015-P01 and HR1015-P01). Compression was tuned by maximizing brightness with a fluorescein sample.
Here, 400-500 mW of $1300 \mathrm{~nm}$ illumination was available after the objective, corresponding to transmission from laser source to sample of $\sim 20 \%$. The maximum field of view of three-photon excitation was $360 \times 360 \mu \mathrm{m}$. Images were acquired with dual linear galvanometers at a frame rate of $\sim 8 \mathrm{~Hz}$.

\section{Illumination intensity}

Photodamage is often a concern in light microscopy. Photodamage can result from linear processes, principally heating (resulting from the absorption of infrared light by water in brain tissue) and from non-linear processes. Non-linear processes are of particular concern with high-energy pulsed sources such as those used for two-photon and three-photon fluorescence microscopy. Heating-related photodamage often occurs with $>250$ $\mathrm{mW}$ of prolonged illumination at $800-1040 \mathrm{~nm}$ (Podgorski and Ranganathan, 2016) and the molar extinction coefficient of water at $1300 \mathrm{~nm}$ is $\sim 2 \times$ that at $900 \mathrm{~nm}$ (Curcio and Petty, 1951; Hale and Querry, 1973; Bertie and Lan, 1996), suggesting that heating-related tissue damage may occur at more than $\sim 100-150 \mathrm{~mW}$ of prolonged illumination at $1300 \mathrm{~nm}$. To avoid damage, we used illumination intensities $<100 \mathrm{~mW}$. Typically, we could image through the depth of neocortex using $<30-\mathrm{mW}$ illumination while maintaining a signal-to-noise ratio comparable to typical two-photon experiments. We rarely observed signs of photodamage, even in mice subjected to $2 \mathrm{~h}$ of continuous three-photon imaging per day for $5 \mathrm{~d}$.

\section{Near-simultaneous two-photon and three-photon excitation}

For two-photon excitation, we used a Coherent Chameleon Ultra II laser source at $920 \mathrm{~nm}$. For nearsimultaneous two-photon and three-photon excitation, we used a Nikon $16 \times / 0.8$ objective $(50 \%$ transmission at $1300 \mathrm{~nm}$ ). Time-averaged power available after the objective was $200-250 \mathrm{~mW}$ at $1300 \mathrm{~nm}$. To match the focal planes of two-photon and three-photon excitation, to the two-photon path, we added an electrically-tunable lens (EL-10-30-TC, Optotune).

\section{Mice and surgeries}

We used Cre-lox transgenic mice to drive GCaMP6s expression in excitatory neurons throughout cortical layers and areas, crossing Emx1-IRES-Cre (B6.129S2-Emx1tm1 (cre)Krj/J, JAX stock number 005628; Gorski et al., 2002) or Slc17a7-

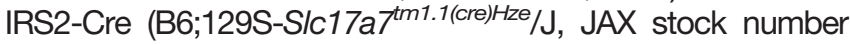
023527; Harris et al., 2014) and Ai162(TIT2L-GCaMP6sICL-tTA2 reporter mice (JAX stock number 031562; Daigle et al., 2018).

A chronic cranial window was implanted over visual cortex as described previously (Goldey et al., 2014; de Vries et al., 2018). Briefly, under $0.5-2 \%$ isoflurane anesthesia, a head restraint bar was attached to the skull using C \& B Metabond (Parkell) and a 5-mm diameter craniotomy was opened over the left visual cortex at coordinates $2.7 \mathrm{~mm}$ lateral, $1.3 \mathrm{~mm}$ anterior to lambda. A durotomy was performed and the craniotomy was sealed with a stack of three \#1 coverslips, attached to each other using 
optical adhesive, and attached to the skull with Metabond.

\section{Visual stimuli}

Visual stimuli were full-field sinusoidal gratings of six orientations, each drifting perpendicular to its orientation (12 directions), at spatial frequencies of 0.04 and 0.08 cycles per degree and a temporal frequency of $1 \mathrm{~Hz}$. Each grating was presented 8 times in random order, each for $2 \mathrm{~s}$ with $1 \mathrm{~s}$ of gray screen between presentations; $0^{\circ}$ corresponds to a grating drifting horizontally in the nasalto-temporal direction and $90^{\circ}$ to a downward-drifting grating. The visual stimulus display and its calibration were as described previously (de Vries et al., 2018). Briefly, stimuli were displayed on an LCD monitor, $15 \mathrm{~cm}$ from the right eye, gamma-corrected, and of mean luminance of $50 \mathrm{~cd} / \mathrm{m}^{2}$. Spherical warping was employed to ensure the apparent size, speed, and spatial frequency were constant across the monitor.

\section{Image analysis}

Image analysis was performed using custom routines in Python 3. For comparison of two-photon and threephoton excitation, images were first separated into twophoton and three-photon movies. Dark current, the mean of several images acquired with no laser illumination, was measured in each movie and subtracted. Image brightness was measured in digitizer units. To avoid artifacts, each movie was normalized to the same mean brightness.

Image contrast was expressed on a scale from 0 (no contrast) to 1. Contrast was calculated locally (in $22.5 \times$ 22.5-pixel blocks) from the temporal mean projection of a movie, the final value being the mean of all the blocks. Contrast in each block was defined as 1 , minimum brightness/maximum brightness.

Each movie was motion-corrected and putative neuronal somata identified by segmentation. Soma and neuropil fluorescence traces were extracted and neuropil fluorescence was subtracted from the corresponding soma trace $(r=1)$. Motion correction, segmentation and trace extraction were performed using Suite2p (Pachitariu et al., 2017) with default settings except for maxregshift which was set to 0.2 to permit less than or equal to $\sim 70-\mu \mathrm{m}$ motion correction in each transverse axis. We manually checked trace extraction for a small sample of neurons by applying spatial masks to motion-corrected fluorescence movies. Neuropil subtraction, calculation of $\Delta F / F$, averaging of traces and identification of direction preference were performed in Python. The preferred direction for each neuron was defined as the direction that evoked the largest mean peak change in $\Delta F / F$.

Motion correction was the mean of $x$ - and $y$-corrections applied by Suite2p. Neuron count was the number of putative somata returned by Suite2p, with manual editing to assist the sorting of somatic from non-somatic regions of interest; \% match was the percentage of putative neurons segmented in the three-photon image that were also segmented in the corresponding two-photon image, assessed manually by comparing images of segmented regions. Pearson correlation coefficient was calculated from the neuropil-subtracted fluorescence traces using scipy.stats.pearsonr. To ensure that the correlation coefficient calculation was from matching regions of interest, traces were extracted from two-photon and three-photon movies using regions of interest segmented from threephoton movies.

To compare two-photon and three-photon measurements of responses to drifting gratings, we used two measures: mean fluorescence response and preferred direction. Again, these measures were applied to traces extracted from two-photon and three-photon movies using regions of interest segmented from three-photon movies.

\section{Experimental design and statistical analysis}

Results supporting Figure 3 were derived from two mice, one male and one female. A total of 1145 regions of interest, corresponding to putative somata, were identified in images from these two mice.

\section{Modeling in-focus and out-of-focus fluorescence}

To estimate the out-of-focus fluorescence generated by excitation light focusing through a homogeneous volume of fluorescent and scattering tissue, we modeled the intensity of ballistic and scattered light, $I_{b}(z, \rho)$ and $l_{s}(z, \rho)$, respectively, in a plane transverse to the optical axis defined by the polar radius, $\rho$, and depth $z$ below the surface of the brain. We calculated the out-of-focus, twophoton-excited fluorescence $\left(F_{\text {oof }}\right)$ numerically, following Theer and Denk (2006),

$$
F_{\text {oof }}=C_{2 p} \int_{V}\left[I_{s}(z, \rho)+I_{b}(z, \rho)\right]^{2} d V,
$$

where $V$ is the out-of-focus illuminated volume of tissue, $C_{2 p}$ is a modality-specific scaling factor incorporating contributions from fluorophore concentration and excitation efficiency, and assumed to be constant over the volume.

We neglected possible depth dependence of fluorescence collection and detection, non-conservative attenuation due to bulk absorption of near-IR light, and the time dependence of excitation by ultrashort pulses that becomes a significant factor for pulse widths less than $\sim 50$ fs (Theer and Denk, 2006; but see also Leray et al., 2007).

Previous models (Xu and Webb, 1996; Theer and Denk, 2006) neglected the difference in distances traveled through tissue by on-axis and marginal rays. The difference in distance can be substantial for high-numerical aperture objectives, but of marginal importance when the focal plane is many multiples of the scattering length below the tissue surface. Here, we calculated fluorescence with the focal plane one to four scattering lengths below the tissue surface and therefore account for the dependence on propagation angle relative to the optical axis by incorporating a radially varying propagation distance,

$$
s(z, \rho)=z \sqrt{1+\frac{\rho^{2}}{\left(z_{0}-z\right)^{2}}},
$$

where $z_{0}$ is the focal plane depth. This factor modifies the intensity profiles of $I_{b}$ and of $I_{s} . F_{\text {oof }}$ can be decomposed 
into individual contributions from ballistic, scattered, and cross-term interaction excitation, for two-photon excitation:

$$
\begin{gathered}
F_{\text {oof }}=\int_{\text {Voof }} d z \int_{A}\left[l_{b}^{2}(z, \rho)+l_{s}^{2}(z, \rho)+2 l_{s}(z, \rho) l_{b}(z, \rho)\right] \\
d A=\int_{\text {Voof }}\left[F_{b}(z)+F_{s}(z)+F_{s b}(z)\right] d z,
\end{gathered}
$$

where, $V_{\text {oof }}$ is the out-of-focus volume denoting the range $\left(-\infty, z_{0}-\delta\right) \cup\left(z_{0}+\delta, \infty\right), \delta$ is the exclusion depth of in-focus light around $z_{0}$.

In our calculations, $\delta$ was a fifth of the scattering length, or $40 \mu \mathrm{m}$, which we assume to be larger than the depth of focus and therefore underestimates the magnitude of the background; wavelength was $900 \mathrm{~nm}$; numerical aperture 0.8 ; and anisotropy factor 0.9 .

To calculate ballistic and scattered light intensities, we considered a Gaussian beam propagating from the surface $(z=0)$ of a scattering medium of scattering length $I=1 /$ a to a ballistic focus located at $z=z_{0}$. We introduced a direction dependent propagation length $s$ $(z, \rho)=z \sqrt{1+\rho^{2} /\left(z_{0}-z\right)^{2}}$, and calculate the ballistic intensity profile at depth $z$ and radial distance $\rho$ according to

$$
I_{b}(z, \rho)=\frac{2 P_{0}}{\pi W^{2}(z)} \exp \left[\frac{-2 \rho^{2}}{w^{2}(z)}\right] \exp [-a s(z, \rho)],
$$

where, $w(z)=2 \sqrt{\lambda\left(\left(z_{0}-z\right)^{2}+z_{R}^{2}\right) / 4 \pi n z_{R}}$ is the $1 / e^{2}$ width, $z_{R}=\lambda / n \pi \tan ^{2} \theta$ is the Rayleigh length determined by the NA-derived focusing half-angle.

As in Theer and Denk (2006), we calculated the intensity distribution of scattered light from a beam spread function derived for small-angle scattering (McLean et al., 1998). We integrated over temporal and angular coordinates to obtain the normalized spatial distribution function

$$
h(z, \rho)=\frac{3 n}{\pi a z^{3}\left\langle\Theta^{2}\right\rangle} \exp \left[-\frac{3 n \rho^{2}}{a z^{3}\left\langle\Theta^{2}\right\rangle}\right] .
$$

The spreading parameter $\left\langle\Theta^{2}\right\rangle=2(1-g)$ is derived from the anisotropy factor $g$ and the function $h(z, \rho)$ accounts for the diffusive spreading of scattered light with increasing depth from an initial on-axis ray, with total power increasing with depth according to $1-\exp [-$ $a z]$, modeling the transfer of energy from the ballistic to the scattered field.

Integrating over the initial surface distribution, the intensity distribution of scattered light at depth $z$ is

$$
\begin{gathered}
l_{s}(z, \rho)=\int_{0}^{2 \pi} d \varphi \int_{0}^{\infty} \frac{2 P_{0} \beta}{\pi^{2} w_{0}^{2}} \exp \left[-\frac{2 \eta^{2}}{w_{0}^{2}}\right] \\
\exp \left[-\beta\left(\rho^{2}+\left(\frac{z_{0}-z}{z_{0}}\right)^{2} \eta^{2}-2 \rho\left(\frac{z_{0}-z}{z_{0}}\right) \eta \cos \varphi\right)\right] \\
\left(1-\exp \left[-a s_{0}\right]\right) \eta d \eta,
\end{gathered}
$$

where, $\beta \equiv 3 n / a s_{0}^{3}\left\langle\Theta^{2}\right\rangle, s_{0}=z \sqrt{1+\eta^{2} / z_{0}^{2}}$ is the propagation distance from the surface, and $w_{0}=$ $2 \sqrt{\lambda\left(z_{0}^{2}+z_{R}^{2}\right) / 4 \pi n z_{R}}$ is the Gaussian beam width at the surface.

\section{Proportion of fluorescence originating from the focal plane}

Calculation of the ratio of in-focus and out-of-focus fluorescence was based on image contrast. We subdivided the $256 \times 512$-pixel images of the motioncorrected, time-averaged two-photon and three-photon movies into $32 \times 32$-pixel subregions. Within each subregion, we determined the minimum pixel value and pixel value mean, $\min _{n}(\bar{F})$ and $\langle\bar{F}\rangle_{n}$, respectively, where $\bar{F}$ denotes the time-averaged fluorescence in each pixel with the minimum and mean functions over the $32 \times 32=$ 1024 pixels. For each subregion in each imaging modality (two-photon and three-photon), we then calculated a contrast parameter, $\gamma_{j, k} \equiv\langle\bar{F}\rangle-\min (\bar{F}) /\langle\bar{F}\rangle$, for the $j$-th subregion in the $k=\{2,3\}$ (two-photon, three-photon) modality.

To calculate in-focus and out-of-focus fluorescence, we made three assumptions. First, we assumed the timeaveraged fluorescence in each pixel reflects the sum of the in-focus and out-of-focus fluorescence $\left(\bar{F}=\bar{F}_{i}+\right.$ $\left.\overline{F_{\text {oof }}}\right)$. Second, we assumed three-photon excitation generates no out-of-focus fluorescence so that $\bar{F}=\bar{F}_{i}$ for three-photon excitation. Third, we assumed in-focus fluorescence is proportional to a modality-independent concentration factor, $C$, with a modality-dependent proportionality constant, so that $F_{j k}=\alpha_{k} C$.

Hence $\gamma_{j, 3 p}=\langle\bar{C}\rangle-\min (\bar{C}) /\langle\bar{C}\rangle$ and $\gamma_{j, 2 p}=\alpha_{2 p}(\langle\bar{C}\rangle-$ $\min (\bar{C})) / \alpha_{2 p}\langle\bar{C}\rangle+F_{\text {oof }}$.

As a measure of the percentage of fluorescence that originates from the focal plane, we calculated an empirical contrast ratio (ECR): ECR $\equiv \gamma_{j, 2 p} / \gamma_{j, 3 p}=\alpha_{2 p}\langle\bar{C}\rangle / \alpha_{2 p}$ $\langle\bar{C}\rangle+F_{\text {oof }}=\bar{F}_{i} / \bar{F}_{i}+\bar{F}_{\text {oof }}$.

The ECR calculated in each subregion was averaged over the subregions to determine the time-averaged ECR for a given imaging depth.

We calculated the theoretical contrast ratio via a signalto-background ratio calculation. We modeled the total in-focus fluorescence, $F_{i}$, according to $F_{i}=\left\langle P_{z 0}\right\rangle^{2} \pi / \lambda$ where $P_{z 0}$ is the total, scattering attenuated, ballistic power estimated at the focal plane according to $P_{z 0}=$ $2 \pi \int_{0}^{\infty} I_{b}\left(z_{0}, \rho\right) \rho d \rho$. The signal-to-background ratio was defined as the ratio of total in-focus to out-of-focus fluorescence, given by $S B R \equiv F_{i} / F_{\text {oof }}$ which ranges from 0 at very large depths to $\infty$ in the background-free case. We defined the contrast ratio, $C R \equiv F_{i} / F_{i}+F_{\text {oof }}=$ $S B R / 1+S B R$ to range from 0 to 1 .

\section{Results}

As an illumination source for three-photon excitation, we used a 40-W Coherent Monaco laser source and Opera- $\mathrm{F}$ optical parametric amplifier, providing $2 \mu \mathrm{J}$, 50 -fs pulses at $1 \mathrm{MHz}$. We configured a MIMMS twophoton microscope for three-photon excitation, exchanging the scan and tube lens to increase transmission 

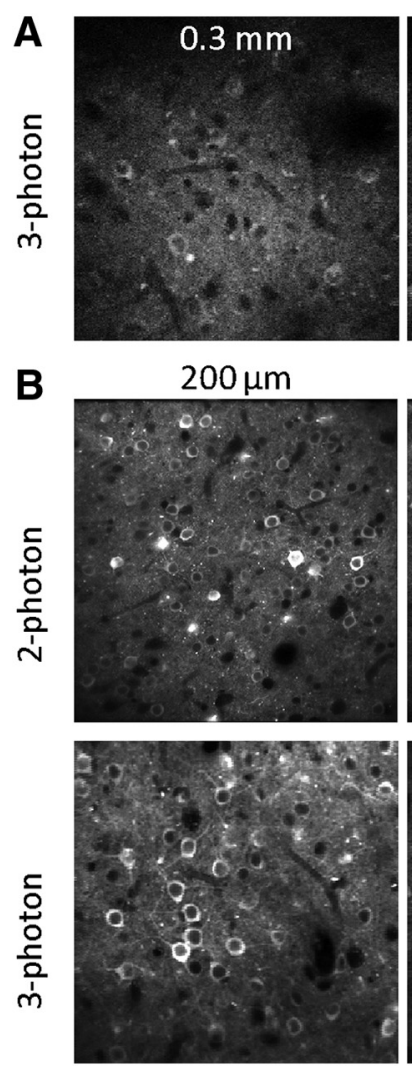
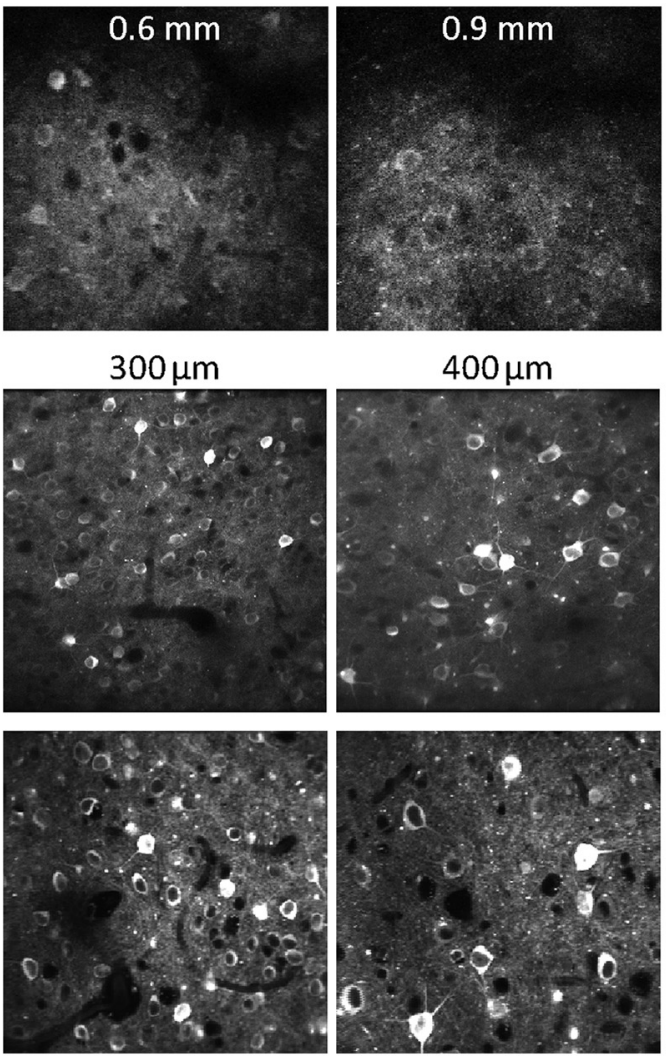
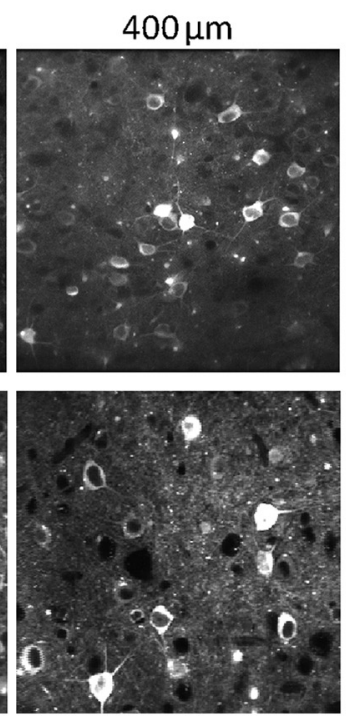

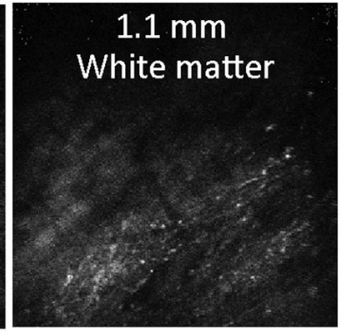

$500 \mu \mathrm{m}$
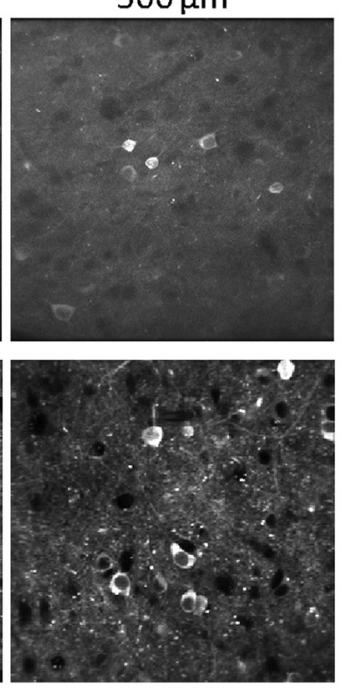

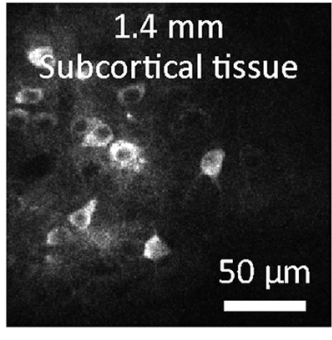

$600 \mu \mathrm{m}$
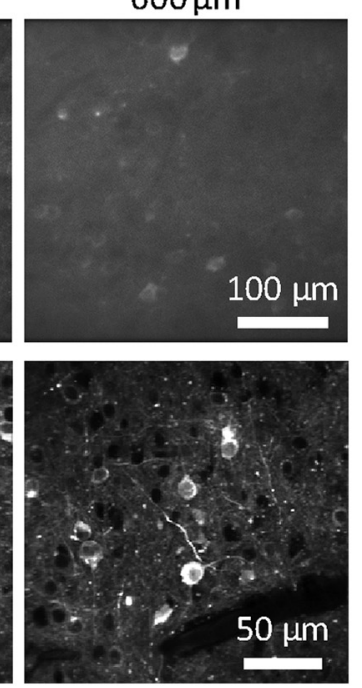

Figure 1. Contrast declines with depth with two-photon excitation. $\boldsymbol{A}$, Example three-photon images from 300, 600, 900, 1100, and $1400 \mu \mathrm{m}$ below the pial surface of visual cortex. Emx1-IRES-Cre;CaMK2a-tTA;Ai94 mouse. B, Comparison of images acquired from a single Emx1-IRES-Cre;CaMK2a-tTA;Ai94 mouse (different fields of view) using two-photon and three-photon excitation, focused 200-600 $\mu \mathrm{m}$ below the pial surface of visual cortex.

through the microscope at $1300 \mathrm{~nm}$ and added a compressor to compensate for pulse dispersion between the laser source and sample. Through a cranial window over visual cortex, we were routinely able to image neurons $>1$ $\mathrm{mm}$ below the pial surface of cortex in GCaMP6 mice (Fig. $1 A)$. Fluorescence intensity followed a cubic relationship with illumination intensity, consistent with fluorescence being driven by the absorption of three photons.

In mice expressing GCaMP broadly in cortical pyramidal neurons, loss of contrast was noticeable in twophoton images from hundreds of micrometers below the brain surface, where contrast was preserved by threephoton excitation (Fig. 1B). To compare two-photon and three-photon excitation more directly, we implemented near-simultaneous two-photon and three-photon excitation. We used two laser sources, combining the beams immediately before the scanning galvanometers (Fig. 2A). With a fast Pockels cell on each laser line acting as a shutter, we alternated two-photon and three-photon excitation, line-by-line (Fig. 2B). The line duration was 0.5 $\mathrm{ms}$, resulting in $0.5 \mathrm{~ms}$ separation of two-photon and three-photon images.

In superficial cortex, two-photon and three-photon results were similar. The same neurons were visible in nearsimultaneous two-photon and three-photon images and changes in fluorescence were coincident in two-photon and three-photon image pairs (Fig. 2C; Movie 1); the results of motion correction and segmentation on twophoton and three-photon movies were similar (SDs of motion correction distributions $<2 \mu \mathrm{m}$ at $<350 \mu \mathrm{m}$; Fig. $3 C)$; there were 50-90 neurons identified in each image (Fig. 3D); $>80 \%$ of neurons in three-photon images matched a neuron in the corresponding two-photon image (Fig. 3E); and traces extracted from matching neurons in two-photon and three-photon movies were strongly correlated, with Pearson correlation coefficients of $\sim 0.8$ 0.9 (Fig. 3F), consistent with previous studies (Wang et al., 2017; Ouzounov et al., 2017, 2019).

The similarity of two-photon and three-photon results declined with depth. In three-photon images, image contrast, motion correction, and number of neurons changed little with depth. In two-photon images, contrast declined incrementally with depth, to near zero at $650 \mu \mathrm{m}$ (Fig. 3B). Lateral motion correction from two-photon movies increased with depth: the SD of motion correction was $<3$ $\mu \mathrm{m}$ at $<400 \mu \mathrm{m}$; at $650 \mu \mathrm{m}$, the SD of lateral motion correction was $<3 \mu \mathrm{m}$ for three-photon excitation and $\sim 25 \mu \mathrm{m}$ for two-photon excitation (Fig. 3C). The segmentation routine identified few neurons in deep locations (Fig. 3D), and the overlap between matching neurons in two-photon and three-photon images and the correlation coefficient between the resulting traces both declined at $>350-400 \mu \mathrm{m}$ (Fig. 3E,F). 
A
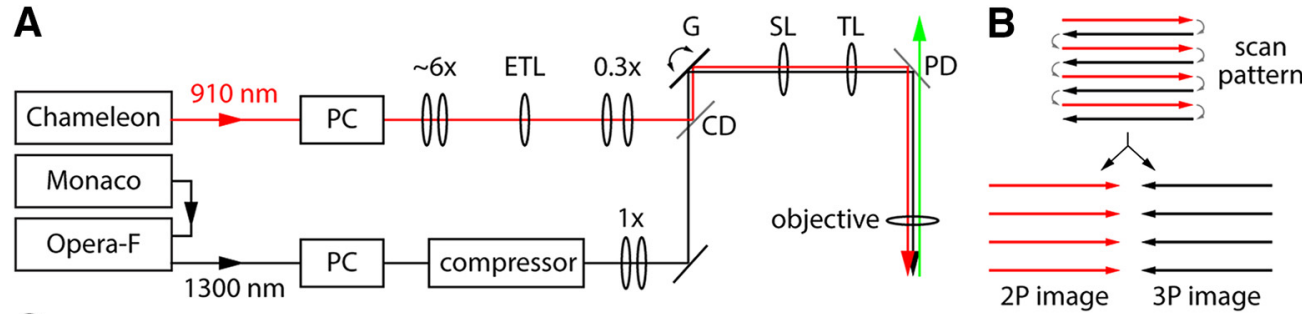

C
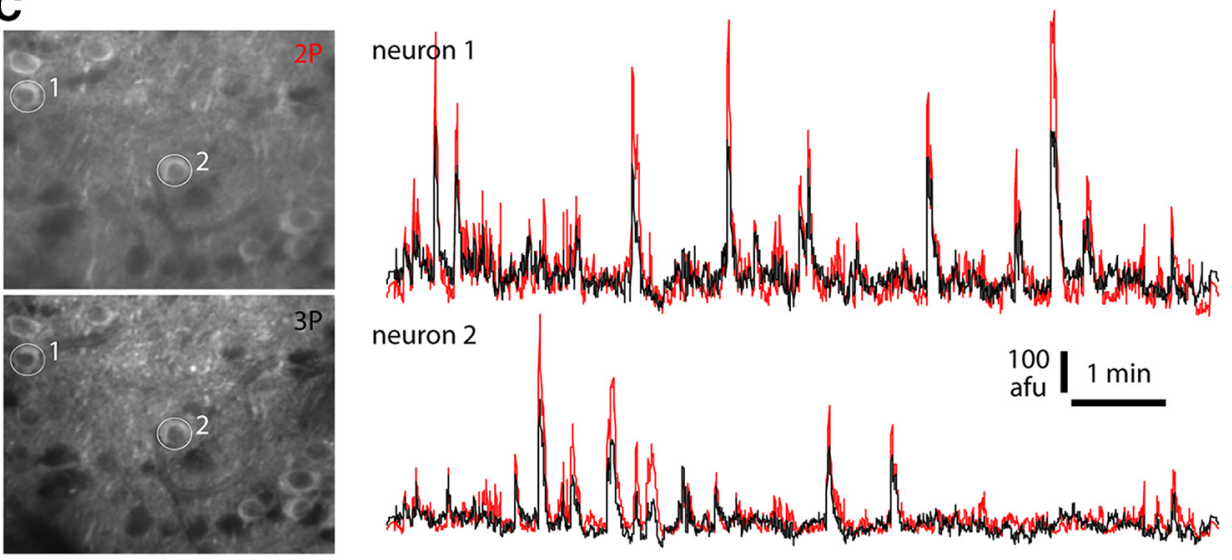

Figure 2. Implementation of near-simultaneous two-photon and three-photon excitation. $\boldsymbol{A}$, Schematic of the optical layout for near-simultaneous two-photon and three-photon excitation; 1300-nm beam (black) passed a Pockels cell (PC), prism compressor, a collimating telescope, combining dichroic mirror (CD), x-y galvanometer pair (G), scan lens (SL), tube lens (TL), FF735-DI02 primary dichroic mirror (PD), and objective lens; 910-nm beam (red) passed a PC, beam expansion to $\sim 1 \mathrm{~cm}$ in diameter, electrically-tunable lens (ETL), $0.3 \times$ beam expansion before being reflected by the combining dichroic mirror onto the galvanometer pair. $\boldsymbol{B}$, Scanning strategy for near-simultaneous two-photon and three-photon excitation. Red: 920-nm excitation, no 1300-nm excitation. Black: no 920-nm excitation, 1300-nm excitation. Gray: both lasers blocked. Lines were sorted into two-photon and three-photon images. $\boldsymbol{C}$, Example images from $350 \mu \mathrm{m}$ below the pia, with traces from two somata.

To determine how the decline in image quality with depth affects the functional properties of cortical neurons measured with two-photon excitation, we examined the apparent responses of cortical neurons to visual stimuli.

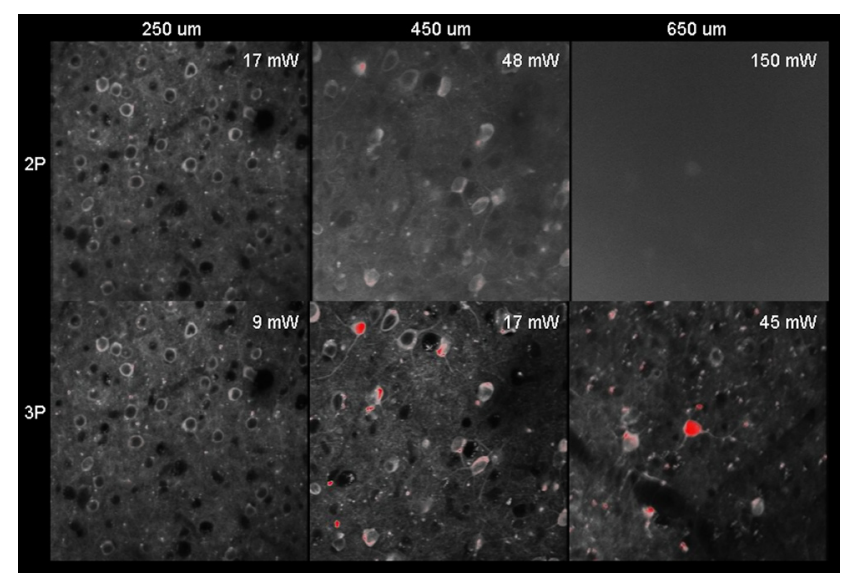

Movie 1. Examples of simultaneous two-photon and three-photon image pairs at different depths. Examples of matched two-photon and three-photon movies 250 , movies 450 , and movies $650 \mu \mathrm{m}$ below the pia. Two-photon and three-photon movie pairs were acquired pseudosimultaneously. Each movie was acquired at a different illumination intensity and each was scaled differently for display purposes. Slc17a7-Cre;Ai162 mouse. Movies acquired at $8 \mathrm{~Hz}$. Playback at 20 $\mathrm{Hz}$. [View online]
We presented sinusoidal gratings drifting in 12 directions and calculated the direction preference of each neuron from extracted fluorescence traces, comparing results from two-photon and three-photon excitation. For superficial neurons, visually-evoked changes in two-photon and three-photon fluorescence were almost identical, trial-by-trial (Fig. 3G) and the resulting preferred direction of each neuron was closely matched (Fig. $3 H$ ), with $83 \%$ (305 of 368) of neurons $\leq 350 \mu \mathrm{m}$ from the brain surface exhibiting identical preferred directions with two-photon and three-photon excitation. The percentage of neurons with matching two-photon and three-photon direction preference declined with depth and at $600 \mu \mathrm{m}$, the number of neurons with matching preference was above chance $(1 / 12=8.3 \%)$, but $\ll 50 \%$.

Two-photon and three-photon excitation produce equivalent results from superficial depths, but the results become less similar $>400 \mu \mathrm{m}$ below the brain surface. Increasing out-of-focus fluorescence and the resulting decline in image contrast are the likely cause. From the ratio of contrast in two-photon and three-photon images, we estimated the percentage of fluorescence that originated from the focal plane during two-photon excitation. As expected, the percentage of two-photon fluorescence originating from the focal plane decreased with increasing depth (Fig. 4A). In-focus and out-of-focus fluorescence were equal at $\sim 400-450 \mu \mathrm{m}$, the depth beyond which the results of two-photon excitation are inaccurate. Hence 
A
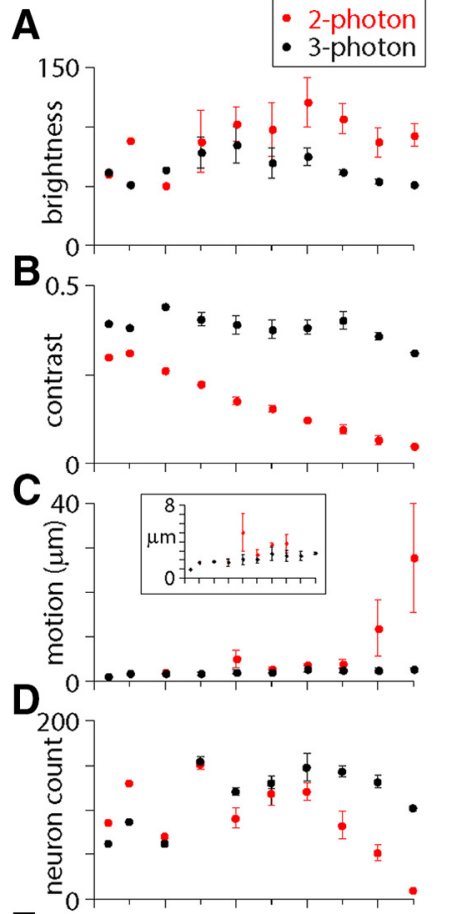

$\mathbf{E}_{10}$

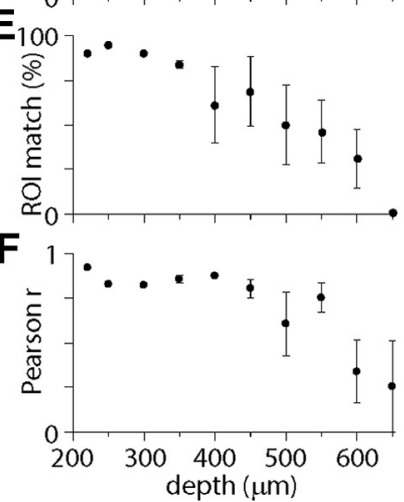

G

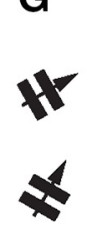

2-photon $350 \mu \mathrm{m}$

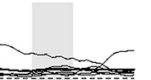

3-photon

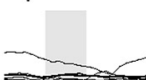

$\Longrightarrow$

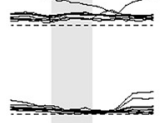

$\pm$
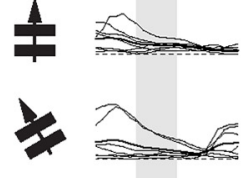

$\ldots$
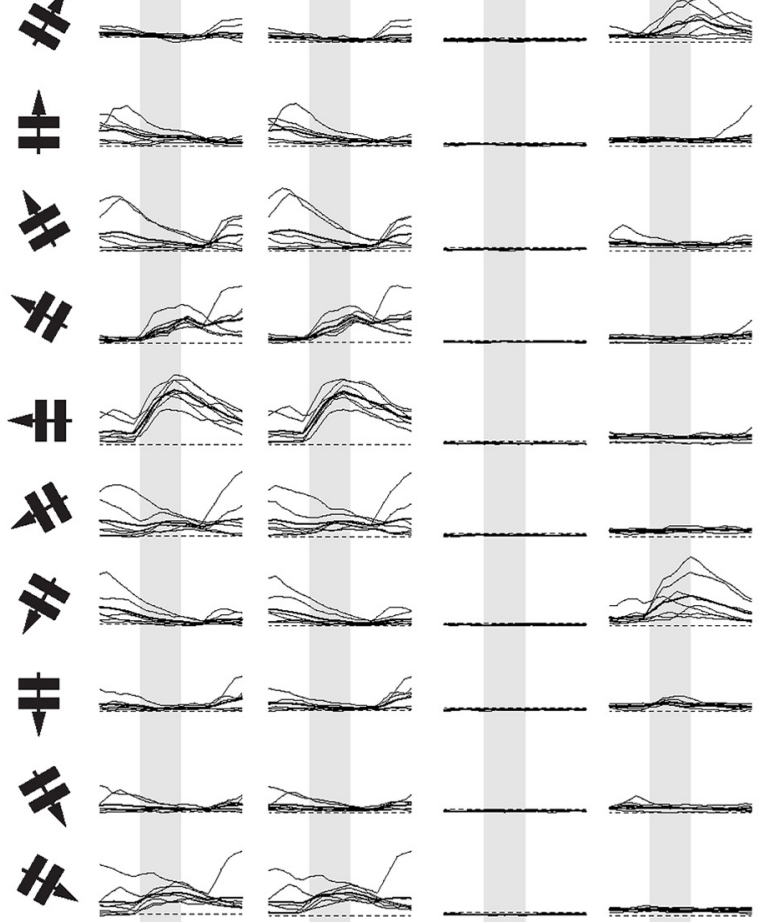

H
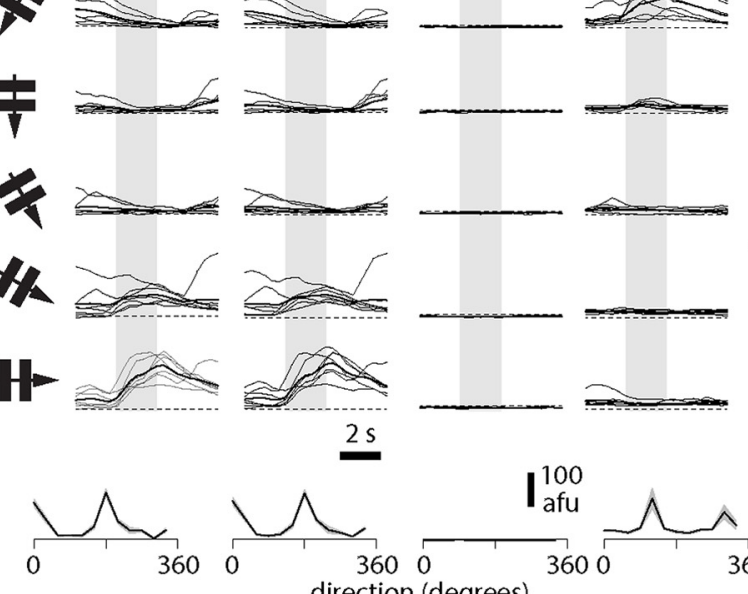

3600

direction (degrees)
H
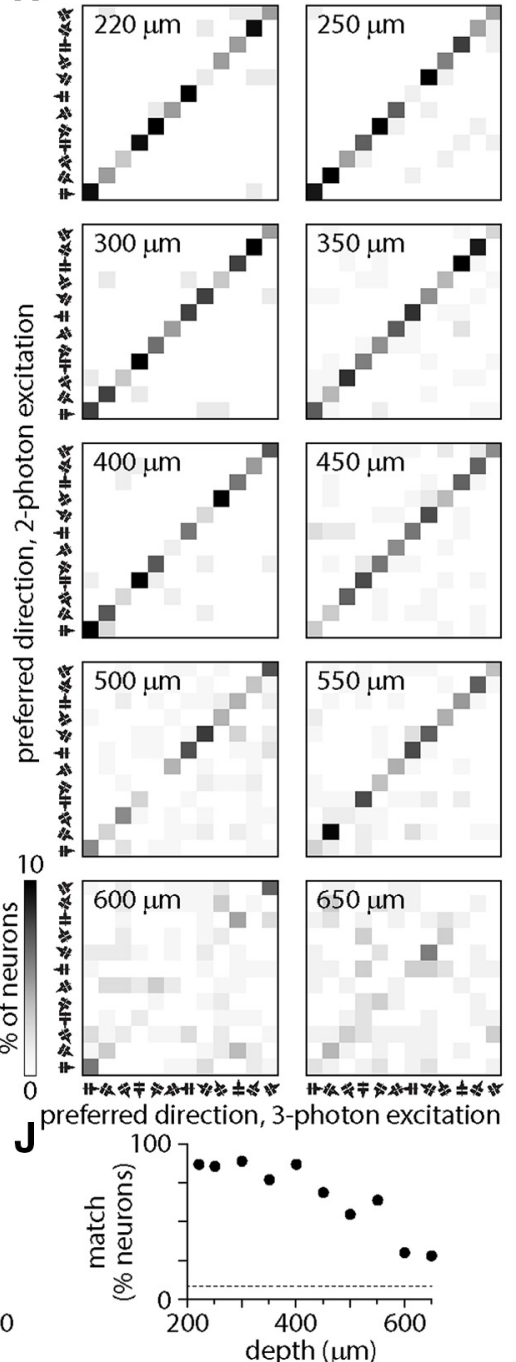

Figure 3. Changes in two-photon image quality and apparent $\Delta \mathrm{F}$ with depth. $\boldsymbol{A}-\boldsymbol{D}$, Plots of image brightness $(\boldsymbol{A})$, contrast $(\boldsymbol{B})$, corrected motion $(\boldsymbol{C})$, and ROI count $(\boldsymbol{D})$ for two-photon (red) and three-photon excitation (black), plot as a function of depth below the pial surface of cortex. Mean \pm SEM of three experiments from two Slc17a7-Cre;Ai162 mice. E, ROI match (percentage of three-photon ROls also segmented from two-photon images) as a function of depth. $\boldsymbol{F}$, Pearson correlation coefficient between two-photon and three-photon fluorescence traces, plot as a function of depth. G, Two-photon and three-photon changes in fluorescence to grating stimuli for two neurons, 350 and $500 \mu \mathrm{m}$ below the pia. Each panel shows change in fluorescence (in arbitrary fluorescence units) through time during presentation of the drifting grating (icon to left indicates orientation and direction) for $2 \mathrm{~s}$ (gray bar). Eight individual traces and the mean (thick line) per direction. Dashed line indicates zero fluorescence. Below, Resulting direction tuning curve. $\boldsymbol{H}$, Plots comparing preferred direction of neurons measured with two-photon $(y$-axis) and three-photon ( $x$-axis) excitation, for each depth. Colors indicate percentages of the total number of neurons at each depth (zero is white, $10 \%$ is black, see color bar). Directions progress at $30^{\circ}$ intervals from the low left corner of each plot (icons). $\boldsymbol{J}$, Percentage of neurons with matching direction preferences measured with two-photon and three-photon excitation, from 200 to $650 \mu \mathrm{m}$. Dashed line: $8.3 \%$.

our results support the depth limit corresponding to the depth at which in-focus and out-of-focus fluorescence are equal.

We compared our measurements of in-focus and outof-focus fluorescence with predictions from theoretical modeling of focused light propagation in scattering tissue (Theer and Denk, 2006). According to this model, 50\% in-focus fluorescence occurs approximately three scattering lengths below the brain surface, at 600-700 $\mu \mathrm{m}$ for a scattering length of $200 \mu \mathrm{m}$ (Fig. 4A). Our measurements indicate that in-focus and out-of-focus fluorescence are equal at $\sim 450 \mu \mathrm{m}$. Three-photon excitation is almost free of out-of-focus fluorescence at these depths.

We expect two-photon excitation to support imaging $>450 \mu \mathrm{m}$ below the brain surface if there are few fluorophore molecules outside the focal plane. Unfortunately, out-of-focus fluorescence arises from fluorophores throughout the tissue above and, to a lesser extent, below the focal plane (Fig. 4B; Theer and Denk, 2006). A reduction in the number of molecules near the brain surface would likely have limited impact on out-of-focus fluorescence and the two-photon depth limit. 

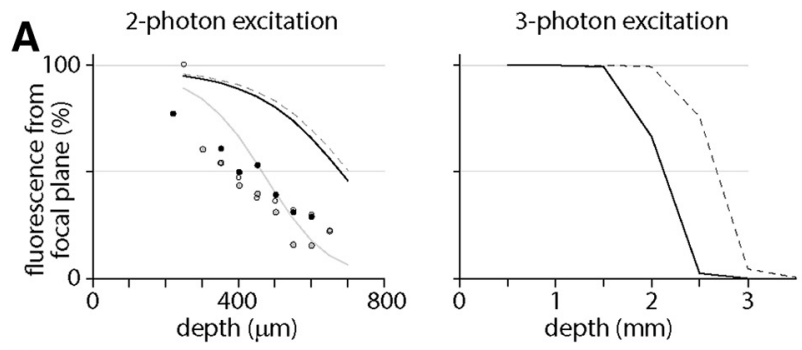

\section{B}
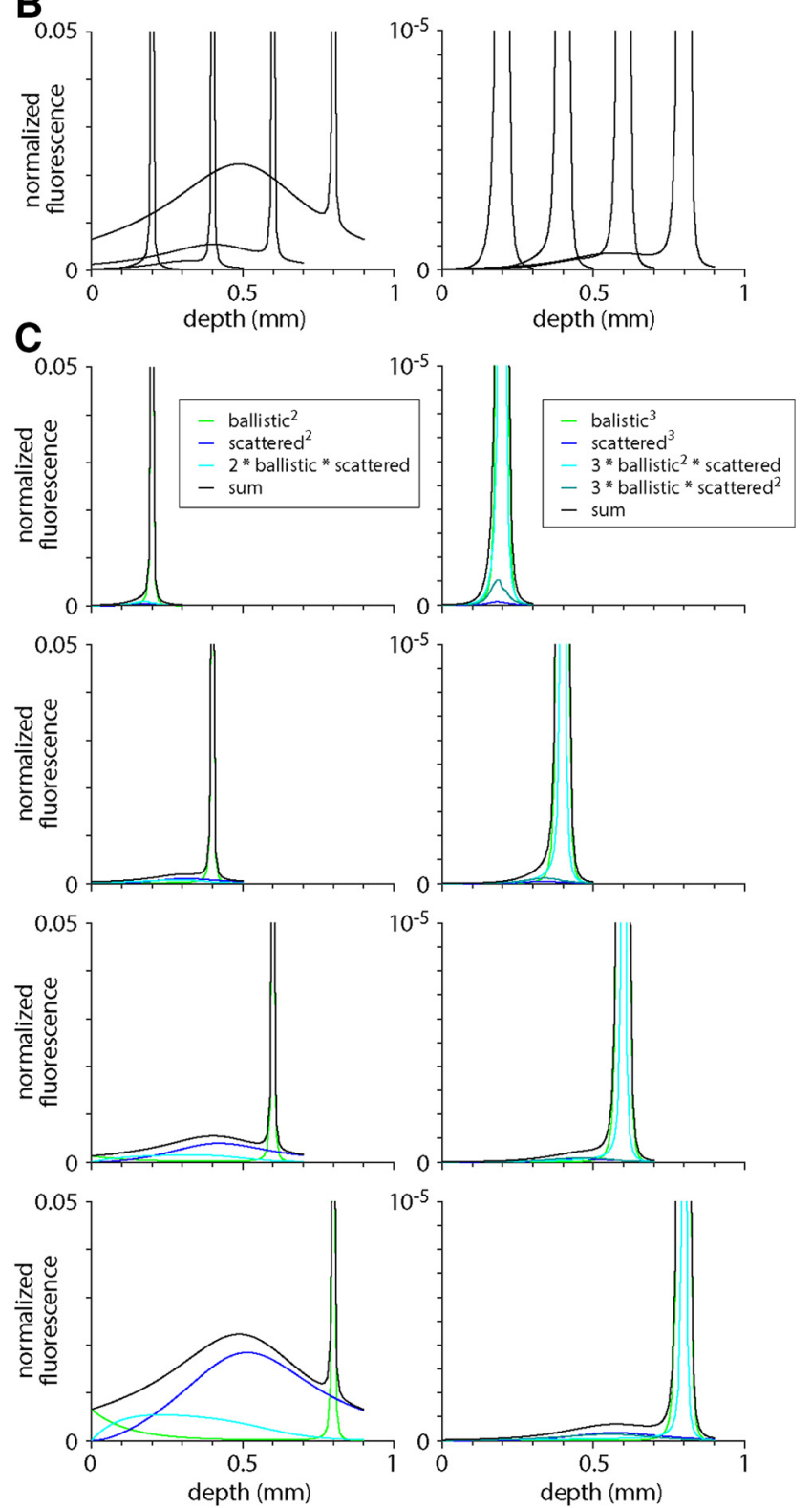

Figure 4. In-focus and out-of-focus fluorescence. $\boldsymbol{A}$, Percentage of total fluorescence that originates from the focal plane, plot as a function of depth of the focal plane below the brain surface. Each point represents a single measurement (from a movie at one depth in one mouse). Lines are calculated from Equation 1 with scattering length constants of $200 \mu \mathrm{m}$ (black) and $150 \mu \mathrm{m}$ (gray). Dashed lines are relationships from the literature for scattering lengths constants of $200 \mu \mathrm{m}$ : Equation 4 of Theer and Denk (2006) for two-photon excitation and Equation 7 of Xu and Webb (1996) for ballistic three-photon excitation. B, Plots show-

\section{Figure 4. continued}

ing the depth from which fluorescence originates with the focal plane at 200, 400, 600, and $800 \mu \mathrm{m}$ below the brain surface. Fluorescence was calculated with Equation 1 and normalized to that in the focal plane. Note the difference in scale for twophoton and three-photon excitation. $\boldsymbol{C}$, Breakdown of sources underlying the total fluorescence in panel $\boldsymbol{B}$ (Theer and Denk, 2006). Two-photon: 900-nm illumination, scattering length 200 $\mu \mathrm{m}$ (Eq. 1). Three-photon: 1300-nm illumination, scattering length $200 \mu \mathrm{m}$, equivalent formulation. Colors: fluorescence from ballistic incident photons (light green), from scattered photons (dark blue) and from a mixture of ballistic and scattered photons (cyan and deep green). Black: the sum of all fluorescence sources (reproduced in panel $\boldsymbol{B}$ ).

\section{Discussion}

We compared the results of two-photon and three-photon excitation of GCaMP6s in excitatory neurons in mouse visual cortex. As expected (Ouzounov et al., 2019), results from superficial cortex were similar, suggesting that neither twophoton nor three-photon images were compromised by saturation or phototoxic effects. With increasing depth from $\sim 250$ to $650 \mu \mathrm{m}$, two-photon image contrast declined and three-photon image contrast was preserved. Many measures (estimated motion, number of neurons segmented, matching of segmented neurons, correlation traces, similarity of fluorescence changes, similarity of preferred direction) were robust to changes in two-photon image contrast to $\sim 400 \mu \mathrm{m}$, but deteriorated between 400 and $550 \mu \mathrm{m}$ on average, some abruptly, compromising measurement of fluorescence changes and direction tuning.

In our experiments, we used a mouse line with GCaMP6s expression in excitatory neurons through all layers of cortex. From the perspective of out-of-focus fluorescence, we expect these mice to be a worst-case scenario for two-photon excitation. In these mice, our results place the depth limit at $\sim 450 \mu \mathrm{m}$ below the brain surface, shallower than the depth predicted by Theer and Denk (2006) and by our calculations. There are several factors that likely contribute to the mismatch of calculations and measurements. Aberrations are present in any imaging system, but not included in our or calculations or those of Theer and Denk (2006). We expect aberrations to reduce the depth at which in-focus and out-of-focus fluorescence are equal. Slight compression of cortex is common in cranial window preparations (de Vries et al., 2018) and might further reduce the depth limit by reducing the scattering length of cortical tissue. Hence one expects the measured depth limit of two-photon excitation to be shallower and our measurements indicate that the depth limit can be as shallow as $\sim 450 \mu \mathrm{m}$.

Our results drive two predictions that we have not tested directly. First, we expect that two-photon excitation will be adequate for characterization of functional properties such as direction tuning in neurons $\leq 450 \mu \mathrm{m}$ from the brain surface in nearly all GCaMP6s mouse lines. Second, we expect two-photon and three-photon results to be comparable at $>450 \mu \mathrm{m}$ in many preparations. We observed substantial mouse-to-mouse variability at 500 $650 \mu \mathrm{m}$, suggesting that two-photon excitation might be 
a viable tool to $>450 \mu \mathrm{m}$ in a small subset of our mice. In other mouse lines and tissues, two-photon excitation at $>450 \mu \mathrm{m}$ will provide more accurate functional measurements in preparations with less out-of-focus fluorescence, including tissues with sparser expression of GCaMP6s and tissues labeled with indicators with low resting fluorescence, such as jGCaMP7c (Dana et al., 2018). In such tissues, out-of-focus fluorescence will be reduced, but will still occur and may equal in-focus fluorescence at a location deeper than $450 \mu \mathrm{m}$. With sufficiently sparse labeling, out-of-focus fluorescence may be insignificant at all depths, in which case the depth limit will likely be set by the thermal limit of brain tissue (Podgorski and Ranganathan, 2016).

There are several strategies that can extend the depth limit of two-photon microscopy. Neuronal structure and activity can be imaged at greater depth with red-shifted fluorophores (Kobat et al., 2009; Tischbirek et al., 2015; Kondo et al., 2017). The increased depth results from reduced scattering of illuminating photons (Wang et al., 2019), which increases intensity in the focal plane and reduces the interaction of scattered photons, the primary source of out-of-focus fluorescence. Aberration correction, such as with adaptive optics, can increase the depth limit. Aberrations are almost inevitable when imaging into intact brain and expand the point spread function (Ji et al., 2010, 2012; Liu et al., 2019), reducing in-focus but not out-of-focus fluorescence. One might expect aberration correction to extend the depth limit toward, but not beyond three scattering length constants, the depth at which in-focus and out-of-focus fluorescence are predicted to be equal in calculations that take no account of aberrations (Theer and Denk, 2006). Finally, reducing the pulse duration to $<20$ fs is expected to increase the in-focus to out-of-focus fluorescence ratio, increasing the depth limit (Theer and Denk, 2006), and where peak pulse energy or heating are limiting factors, more modest changes in pulse duration can increase imaging depth (Theer et al., 2003; Mittmann et al., 2011). Simply increasing illumination intensity, however, will increase in-focus and out-of-focus fluorescence equally, leaving the depth limit unaffected.

In summary, we have established that two-photon and three-photon excitation are equivalent less than or equal to $\sim 450 \mu \mathrm{m}$ below the brain surface in mice with GCaMP6s expression throughout cortical layers. Tentatively, we suggest the depth limit of two-photon excitation is $450 \mu \mathrm{m}$ or deeper in nearly all mouse lines, since few if any mice express a higher proportion of fluorophore molecules outside the focal plane than mice with expression throughout the cortical layers. In tissues with and tissues without extensive fluorophore expression outside the focal plane, three-photon excitation enables measurement of cellular activity beyond the depth limit of two-photon excitation.

\section{References}

Bertie JE, Lan Z (1996) Infrared intensities of liquids XX: the intensity of the $\mathrm{OH}$ stretching band of liquid water revisited, and the best current values of the optical constants of $\mathrm{H}_{2} \mathrm{O}(\mathrm{I})$ at $25^{\circ} \mathrm{C}$ between 15,000 and $1 \mathrm{~cm}^{-1}$. Appl Spectrosc 50:1047-1057.
Curcio JA, Petty CC (1951) The near infrared absorption spectrum of liquid water. J Opt Soc Am 41:302-304.

Daigle TL, Madisen L, Hage TA, Valley MT, Knoblich U, Larsen RS, Takeno MM, Huang L, Gu H, Larsen R, Mills M, Bosma-Moody A, Siverts LA, Walker M, Graybuck LT, Yao Z, Fong O, Nguyen TN, Garren E, Lenz GH, et al. (2018) A suite of transgenic driver and reporter mouse lines with enhanced brain-cell-type targeting and functionality. Cell 174:465-480.

Dana H, Sun Y, Mohar B, Hulse B, Hasseman JP, Tsegaye G, Tsang A, Wong A, Patel R, Macklin JJ, Chen Y, Konnerth A, Jayaraman V, Looger LL, Schreiter ER, Svoboda K, Kim DS (2018) High-performance GFP-based calcium indicators for imaging activity in neuronal populations and microcompartments. bioRxiv 434589.

de Vries SEJ, Lecoq J, Buice MA, Groblewski PA, Ocker GK, Oliver M, Feng D, Cain N, Ledochowitsch P, Millman D, Roll K, Garrett M, Keenan T, Kuan L, Mihalas S, Olsen S, Thompson C, Wakeman W, Waters J, Williams D, et al. (2018) A large-scale, standardized physiological survey reveals higher order coding throughout the mouse visual cortex. bioRxiv 359513.

Goldey GJ, Roumis DK, Glickfeld LL, Kerlin AM, Reid RC, Bonin V, Schafer DP, Andermann ML (2014) Removable cranial windows for long-term imaging in awake mice. Nat Protoc 9:2515-2538.

Gorski JA, Talley T, Qiu M, Puelles L, Rubenstein JL, Jones KR (2002) Cortical excitatory neurons and glia, but not GABAergic neurons, are produced in the Emx1-expressing lineage. J Neurosci 22: 6309-6314.

Hale GM, Querry MR (1973) Optical constants of water in the 200-nm to 200-microm wavelength region. Appl Opt 12:555-563.

Harris JA, Hirokawa KE, Sorensen SA, Gu H, Mills M, Ng LL, Bohn P, Mortrud M, Ouellette B, Kidney J, Smith KA, Dang C, Sunkin S, Bernard A, Oh SW, Madisen L, Zeng H (2014) Anatomical characterization of Cre driver mice for neural circuit mapping and manipulation. Front Neural Circuits 8:76.

Horton NG, Wang K, Kobat D, Clark CG, Wise FW, Schaffer CB, Xu C (2013) In vivo three-photon microscopy of subcortical structures within an intact mouse brain. Nat Photonics 7:205-209.

Ji N, Milkie DE, Betzig E (2010) Adaptive optics via pupil segmentation for high-resolution imaging in biological tissues. Nat Methods 7:141-147.

Ji N, Sato TR, Betzig E (2012) Characterization and adaptive optical correction of aberrations during in vivo imaging in the mouse cortex. Proc Natl Acad Sci USA 109:22-27.

Kobat D, Durst ME, Nishimura N, Wong AW, Schaffer CB, Xu C (2009) Deep tissue multiphoton microscopy using longer wavelength excitation. Opt Express 17:13354-13364.

Kobat D, Horton NG, Xu C (2011) In vivo two-photon microscopy to 1.6-mm depth in mouse cortex. J Biomed Opt 16:106014.

Kondo M, Kobayashi K, Ohkura M, Nakai J, Matsuzaki M (2017) Two-photon calcium imaging of the medial prefrontal cortex and hippocampus without cortical invasion. Elife 6:e26839.

Leray A, Odin C, Huguet E, Amblard F, Le Grand Y (2007) Spatially distributed two-photon excitation fluorescence in scattering media: experiments and time-resolved Monte Carlo simulations. Opt Commun 272:269-278.

Liu R, Li Z, Marvin JS, Kleinfeld D (2019) Direct wavefront sensing enables functional imaging of infragranular axons and spines. Nat Methods 16:615-618.

McLean JW, Freeman JD, Walker RE (1998) Beam spread function with time dispersion. Appl Opt 37:4701-4711.

Mittmann W, Wallace DJ, Czubayko U, Herb JT, Schaefer AT, Looger LL, Denk W, Kerr JND (2011) Two-photon calcium imaging of evoked activity from L5 somatosensory neurons in vivo. Nat Neurosci 14:1089-1094.

Ouzounov DG, Wang T, Wang M, Feng DD, Horton NG, CruzHernández JC, Cheng YT, Reimer J, Tolias AS, Nishimura N, Xu C (2017) In vivo three-photon imaging of activity of GCaMP6-labeled neurons deep in intact mouse brain. Nat Methods 14:388-329.

Ouzounov DG, Wang T, Wu C, Xu C (2019) GCaMP6 $\Delta$ F/F dependence on the excitation wavelength in 3-photon and 2-photon 
microscopy of mouse brain activity. Biomed Opt Express 10:33433352.

Pachitariu M, Stringer C, Dipoppa M, Schröder M, Rossi LF, Dalgleish H, Carandini M, Harris KD (2017) Suite2p: beyond 10,000 neurons with standard two-photon microscopy. bioRxiv 061507.

Podgorski K, Ranganathan G (2016) Brain heating induced by nearinfrared lasers during multiphoton microscopy. J Neurophysiol 116:1012-1023.

Theer P, Denk W (2006) On the fundamental imaging-depth limit in two-photon microscopy. J Opt Soc Am A 23:3139-3149.

Theer P, Hasan MT, Denk W (2003) Two-photon imaging to a depth of 1000 microm in living brains by use of a Ti:Al2O3 regenerative amplifier. Opt Lett 28:1022-1024.

Tischbirek C, Birkner A, Jia H, Sakmann B, Konnerth A (2015) Deep two-photon brain imaging with a red-shifted fluorometric $\mathrm{Ca}+$ indicator. Proc Natl Acad Sci USA 112:11377-11382.
Wang M, Kim M, Xia F, Xu C (2019) Impact of the emission wavelengths on in vivo multiphoton imaging of mouse brains. Biomed Opt Express 10:1905-1918.

Wang T, Ouzounov DG, Wang M, Xu C (2017) Quantitative comparison of two-photon and three-photon activity imaging of GCaMP6s-labeled neurons in vivo in the mouse brain. Optics in the Life Sciences Congress. Optical Society of America, paper BrM4B.4.

Xu C, Webb WW (1996) Measurement of two-photon excitation cross sections of molecular fluorophores with data from 690 to $1050 \mathrm{~nm}$. J Opt Soc Am B 13:481-491.

Yildirim M, Sugihara H, So PTC, Sur M (2019) Functional imaging of visual cortical layers and subplate in awake mice with optimized three photon microscopy. Nat Commun 10:177.

Ying J, Liu F, Alfano RR (1999) Spatial distribution of two-photon-excited fluorescence in scattering media: erratum. Appl Opt 38:2151. 